\title{
Baseline global longitudinal strain predictive of anthracycline-induced cardiotoxicity
}

Raquel Araujo-Gutierrez ${ }^{1}$, Kalyan R. Chitturi ${ }^{1,2}$, Jiaqiong X $\mathrm{u}^{1,3}$, Yuanchen Wang ${ }^{1}$, Elizabeth Kinder ${ }^{1}$, Alpana Senapati ${ }^{1}$, L. Bindu Chebrolu', Mahwash Kassi ${ }^{1}$ and Barry H. Trachtenberg ${ }^{1 *}$

\begin{abstract}
Background: Cancer therapy-related cardiac dysfunction (CTRD) is a major source of morbidity and mortality in long-term cancer survivors. Decreased GLS predicts decreased left ventricular ejection fraction (LVEF) in patients receiving anthracyclines, but knowledge regarding the clinical utility of baseline GLS in patients at low-risk of (CTRD) is limited.
\end{abstract}

Objectives: The purpose of this study was to investigate whether baseline echocardiographic assessment of global longitudinal strain (GLS) before treatment with anthracyclines is predictive of (CTRD) in a broad cohort of patients with normal baseline LVEF.

Methods: Study participants comprised 188 patients at a single institution who underwent baseline 2-dimensional (2D) speckle-tracking echocardiography before treatment with anthracyclines and at least one follow-up echocardiogram 3 months after chemotherapy initiation. Patients with a baseline LVEF $<55 \%$ were excluded from the analysis. The primary endpoint, (CTRD), was defined as an absolute decline in LVEF $>10 \%$ from baseline and an overall reduced LVEF $<50 \%$. Potential and known risk factors were evaluated using univariable and multivariable Cox proportional hazards regression analysis.

Results: Twenty-three patients (12.23\%) developed (CTRD). Among patients with (CTRD), the mean GLS was $-17.51 \% \pm 2.77 \%$. The optimal cutoff point for (CTRD) was $-18.05 \%$. The sensitivity was 0.70 and specificity was 0.70 . The area under ROC curve was 0.70 . After adjustment for cardiovascular and cancer therapy related risk factors, GLS or decreased baseline GLS $\geq-18 \%$ was predictive of (CTRD) (adjusted hazards ratio 1.17, 95\% confidence interval $1.00,1.36 ; p=0.044$ for GLS, or hazards ratio $3.54 ; 95 \%$ confidence interval 1.34, 9.35; $p=0.011$ for decreased GLS), along with history of tobacco use, pre-chemotherapy systolic blood pressure, and cumulative anthracycline dose.

Conclusions: Baseline GLS or decreased baseline GLS was predictive of (CTRD) before anthracycline treatment in a cohort of cancer patients with a normal baseline LVEF. This data supports the implementation of strain-protocol echocardiography in cardio-oncology practice for identifying and monitoring patients who are at elevated risk of (CTRD).

Keywords: Global longitudinal strain, echocardiography, speckle tracking, anthracycline, cardiomyopathy

\footnotetext{
* Correspondence: btrachtenberg@houstonmethodist.org

'Houston Methodist DeBakey Heart and Vascular Center, 6550 Fannin St.,

Suite 1901, Houston, Texas 77030, USA

Full list of author information is available at the end of the article
}

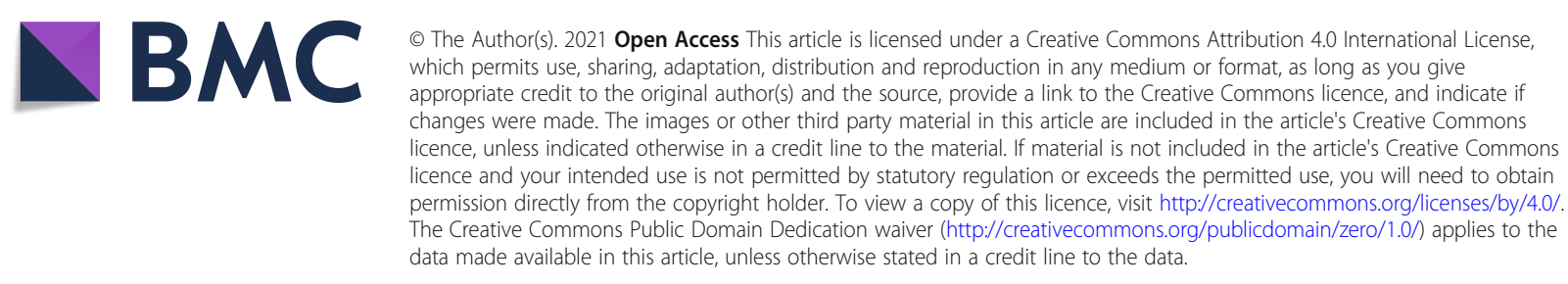




\section{Introduction}

Routinely used in the treatment of hematologic malignancies and solid tumors, anthracyclines can lead to cancer therapy-related cardiac dysfunction (CTRD) in approximately $9 \%$ of patients and is mostly diagnosed within the first year of treatment in patients who are monitored prospectively. Known risk factors include high cumulative dose (i.e. doxorubicin $\geq 250 \mathrm{mg} / \mathrm{m}^{2}$ ) or any dose combined with radiation therapy in the heart field, sequential use of HER 2? her-2 antagonists, age> 60 years, cardiovascular risk factors, and compromised cardiac function (e.g. $\mathrm{LVEF}<55 \%$, pre-existing ischemic or valvular disease) [1].

However, certain patients lacking these high-risk features develop anthracycline-induced cardiomyopathy. Earlier detection of subclinical myocardial dysfunction may identify these individuals, allowing for closer clinical monitoring during and after anthracycline therapy and prior to initiation of potentially cardioprotective interventions.

Two-dimensional speckle tracking echocardiography (STE) with global longitudinal strain (GLS) has become a well-established and important tool to predict subsequent CTRD (commonly defined as a decreased left ventricular ejection fraction (LVEF) $<50$ or $53 \%$ with $\geq 5 \%$ absolute reduction in symptomatic patients or $\geq 10 \%$ in asymptomatic patients) during anthracycline therapy, even in patients without risk factors for CTRD [2-6]. Recent retrospective studies of patients with hematological malignancies show that baseline GLS before initiation of anthracycline therapy is predictive of left ventricular systolic dysfunction or major adverse cardiac events, including cardiac death or symptomatic heart failure [7-9]. However, these studies included patients with borderline LVEF (50-55\%). Our study objective was to evaluate the clinical utility of STE and baseline GLS in detecting CTRD in a broad cohort of cancer patients with a normal LVEF.

\section{Methods}

The study was conducted at Houston Methodist Hospital with patients selected from the Houston Methodist Oncologic Pharmacy Registry, which includes cancerrelated therapy information from the Houston Methodist Cancer Center and 7 hospitals and affiliated cancer centers. The institutional review board of Houston Methodist approved the protocol.

\section{Study population and pre-specified risk factors}

Patients who had a baseline STE within 3 months before the initiation of anthracycline therapy and at least one follow-up echocardiogram 3 months after start of treatment from July 1, 2013, and July 1, 2019 were identified. Patients with a suboptimal strain study (impaired regional tracking in $>2$ myocardial segments), an abnormal left ventricular ejection fraction (LVEF) $<50 \%$, left bundle branch block, history of coronary artery bypass grafting (CABG), premature ventricular contractions, or a systolic blood pressure $>180 \mathrm{mmHg}$ or diastolic blood pressure $>100 \mathrm{mmHg}$ before treatment were excluded (Fig. 1). Each chart was accessed for baseline

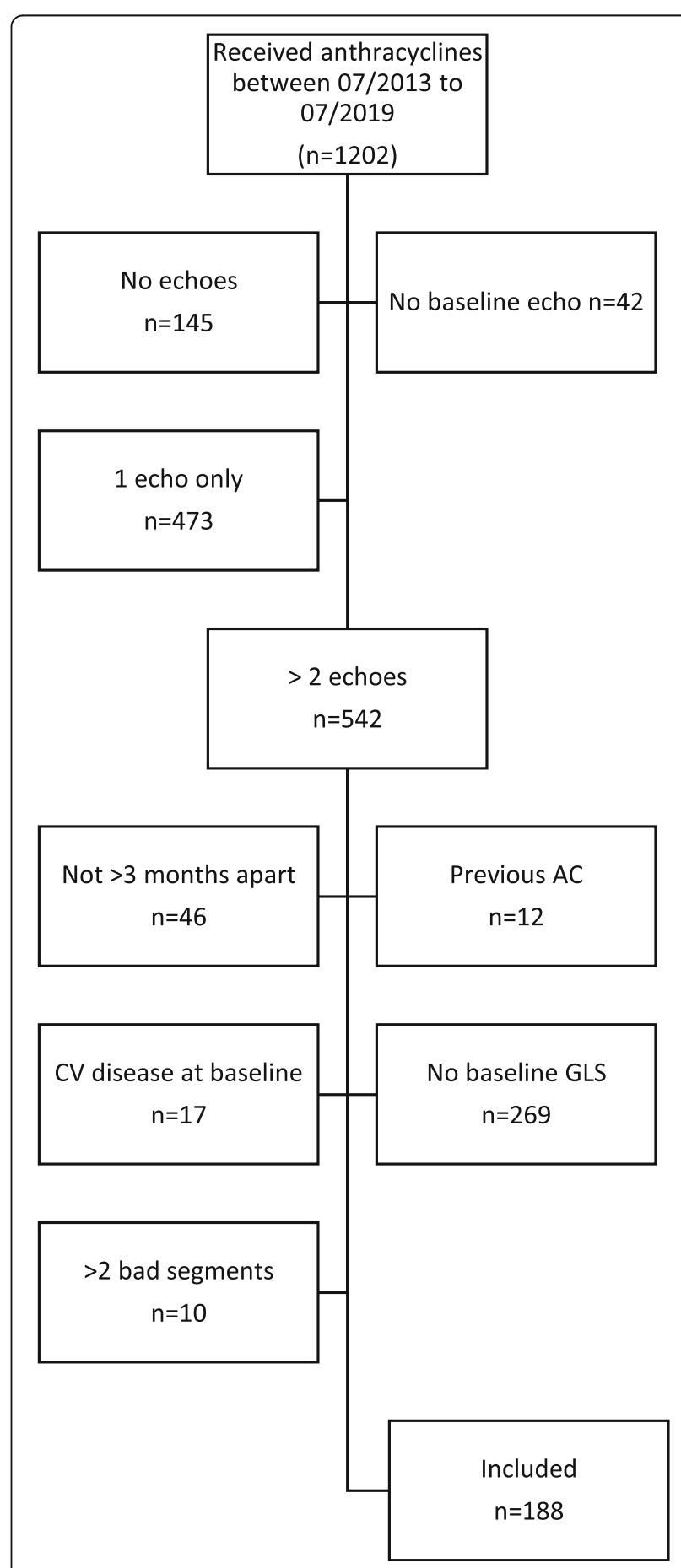

Fig. 1 Patient Selection Flow Chart 
demographics (including age, vitals, cardiovascular (CV) risk factors, medical comorbidities), echocardiography studies, and clinical outcomes (symptomatic heart failure, CV mortality, and non-CV death).

Pre-existing cardiovascular risk factors and medical comorbidities extracted from the electronic medical records, included: body mass index (BMI), tobacco use, diabetes, hypertension, hyperlipidemia, coronary artery disease, hypothyroidism, and a family history of atherosclerotic cardiovascular disease. Cumulative anthracycline dose was calculated based on the following rapid infusion doxorubicin CTRD equivalence: epirubicin, 0.66; daunorubicin, 0.75; and idarubicin, 0.53 [10, 11].

\section{Echocardiography strain protocol and endpoints}

The primary endpoint of the study was CTRD, defined as an LVEF $<50 \%$ and absolute decline $>10 \%$ in LVEF from baseline echocardiography. LVEF was calculated using quantitative (modified Simpson's biplane method) and/or visual analysis of LVEF according to the American Society of Echocardiography standards [12].

The vendors utilized in the strain analysis included the most recent upgraded versions of GE EchoPAC and TomTec Image-Arena. The endocardial borders were traced from 3 apical viewers. Global longitudinal strain was calculated by measuring the entire endocardial line length at the end-diastole and end-systole in each view and averaging the results from the 3 views. Each measurement was taken from the average of 3 consecutive cardiac cycles. The strain quality was assessed objectively by a second observer as bad if more than two segments were un-visualized.

Secondary end points of all-cause mortality and CV mortality were assessed between the patients with normal and decreased baseline GLS. Decreased baseline GLS was defined as $\geq-18 \%$ [13].

\section{Statistical analysis}

Baseline characteristics were summarized according to CTRD status and baseline GLS status (abnormal vs normal). All data were presented as mean \pm SD for continuous variables and number and \% for categorical variables. Chi-square or Fisher's exact test for categorical variables and t-test or Mann-Whitney test for continuous variables were used to determine association of pretreatment variables with decreased baseline GLS and CTRD. The normality assumption was tested by the Shapiro -Wilk tests.

Kaplan-Meier survival curves for overall mortality was calculated in strata defined by decreased vs. normal GLS. Time-on-study was used as time scale in all survival analyses: Time begins at first anthracycline dose and ends in date of death or date of last follow-up. Twosided log rank tests defined significance. The overall survival at 1-year, 3-year, and 5-year intervals between decreased and normal GLS were compared with the pseudo-value approach [12]. Cumulative incidence of cardiovascular related mortality was calculated after adjusted for the competing risk of non-cardiovascular death. Univariable and multivariable Cox proportional hazards regression models were used to examine associations between demographics and clinical variables with CTRD. The multivariable model included baseline GLS and variables selected from backward elimination with the significance level for removal from the model of 0.05 . The full model included all statistically significant variables with $p$-value $<0.05$ in the univariable models. Subsequently, the same multivariable analysis was done when GLS modeled as a categorical variable (decreased vs. normal). The Cox proportionality assumption was verified by including time-dependent interactions of covariates with survival time in the models. There were no violations of this assumption in all models. The Hosmer and Lemeshow type goodness-of-fit statistics for the Cox proportional hazards model was used to check the model fitting [14].

All analyses were performed with STATA version 16 (StataCorp. 2019. Stata Statistical Software: Release 16. College Station, TX: StataCorp LLC). Statistical significance was defined as two-tailed $p<0.05$ for all tests.

\section{Results \\ CTRD vs normal}

A total of 188 patients were included in our study. Twenty-three patients (12.23\%) had CTRD during their treatment course. Baseline characteristics are presented by CTRD in Table 1 . The mean baseline GLS of patients who experienced cardiotoxicity CTRD during treatment with anthracyclines significantly differed from the mean baseline GLS of patients who did not have CTRD $(-17.51 \% \pm 2.77 \%$ vs $-19.36 \% \pm 2.86 \% ; p=0.004)$. Patients in the CTRD group were more likely to be male $(52.17 \%$ vs $30.30 \%, p=0.056)$ and were older $(60.87 \pm 10.89$ vs $52.92 \pm 14.59, p=0.013)$. A history of hypertension was more common in the CTRD group $(69.57 \%$ vs $38.79 \%$, $p=0.007)$ and CTRD patients also had higher baseline systolic blood pressure $(137.74 \pm 23.86$ vs $128.22 \pm 16.35$, $p=0.015)$. In addition, patients with CTRD were more likely to be treated for a hematologic malignancy (78.26\% vs $49.09 \%, p=0.018)$. Although within normal values, LVEF was significantly lower in the CTRD group $(61.7 \pm 4.60$ vs $64.43 \pm 3.73, p=0.002)$. Cumulative anthracycline dose was lower in the CTRD group $\left(118.02 \mathrm{mg} / \mathrm{m}^{2} \pm 93.32 \mathrm{mg} / \mathrm{m}^{2}\right.$ vs $175.62 \mathrm{mg} / \mathrm{m}^{2} \pm 102.49$ $\left.\mathrm{mg} / \mathrm{m}^{2}, p=0.013\right)$. However, there was no significant difference in the total number of cycles in patients with CTRD vs controls, when grouping by breast cancer vs all 
Table 1 Baseline characteristic by cardiotoxicity (defined as drop EF>10\% from baseline and EF $<50 \%$ at each time measurement)

\begin{tabular}{|c|c|c|c|c|}
\hline & Total & Cardiotoxicity & & $P-$ \\
\hline & & $\begin{array}{l}\text { No } \\
N=165\end{array}$ & $\begin{array}{l}\text { Yes } \\
N=23\end{array}$ & \\
\hline Age (years) & $53.89 \pm 14.40$ & $52.92 \pm 14.59$ & $60.87 \pm 10.89$ & 0.013 \\
\hline Male & $62(32.98)$ & $50(30.3)$ & $12(52.17)$ & 0.056 \\
\hline BMI $\left(\mathrm{kg} / \mathrm{m}^{2}\right)$ & $28.40 \pm 6.08$ & $28.40 \pm 6.20$ & $28.44 \pm 5.19$ & 0.98 \\
\hline Race & & & & 0.40 \\
\hline Asian & $5(2.66)$ & $5(3.03)$ & $0(0.00)$ & \\
\hline Black & $24(12.77)$ & $21(12.73)$ & $3(13.04)$ & \\
\hline Caucasian & $99(52.66)$ & $84(50.91)$ & $15(65.22)$ & \\
\hline Hispanic & $50(26.60)$ & $47(28.48)$ & $3(13.04)$ & \\
\hline Other & $10(5.32)$ & $8(4.85)$ & $2(8.70)$ & \\
\hline Systolic & $129.42 \pm 17.68$ & $128.22 \pm 16.35$ & $137.74 \pm 23.86$ & 0.015 \\
\hline Diastolic & $70.97 \pm 10.66$ & $70.67 \pm 10.62$ & $73.09 \pm 10.93$ & 0.31 \\
\hline Heart Rate & $76.38 \pm 13.31$ & $76.23 \pm 13.28$ & $77.53 \pm 13.85$ & 0.69 \\
\hline Family history of Heart Disease & $19(10.11)$ & $15(9.09)$ & $4(17.39)$ & 0.26 \\
\hline Diabetes & $35(18.62)$ & $29(17.58)$ & $6(26.09)$ & 0.39 \\
\hline Hypertension & $80(42.55)$ & $64(38.79)$ & $16(69.57)$ & 0.007 \\
\hline Hyperlipidemia & $52(27.66)$ & $44(26.67)$ & $8(34.78)$ & 0.46 \\
\hline Coronary Artery Disease & $9(4.79)$ & $8(4.85)$ & $1(4.35)$ & 1.00 \\
\hline Hypothyroidism & $21(11.17)$ & $20(12.12)$ & $1(4.35)$ & 0.48 \\
\hline Smoking & $48(25.53)$ & $33(20.00)$ & $15(65.22)$ & $<0.001$ \\
\hline Ejection Fraction & $64.09 \pm 3.94$ & $64.43 \pm 3.73$ & $61.70 \pm 4.60$ & 0.002 \\
\hline Baseline GLS & $-19.13 \pm 2.91$ & $-19.36 \pm 2.86$ & $-17.51 \pm 2.77$ & 0.004 \\
\hline Cancer type & & & & 0.018 \\
\hline Breast & $80(42.55)$ & $76(46.06)$ & $4(17.39)$ & \\
\hline Hematologic & 99 (52.66) & $81(49.09)$ & $18(78.26)$ & \\
\hline Other & $9(4.79)$ & $8(4.85)$ & $1(4.35)$ & \\
\hline Chemotherapy dose & $168.70 \pm 102.91$ & $175.62 \pm 102.49$ & $118.02 \pm 93.32$ & 0.013 \\
\hline
\end{tabular}

Data were presented as mean \pm SD for continuous variables and number (\%) for categorical variables. Chi-square or Fisher's exact test (categorical variables) and ttest or Mann-Whitney test (continuous variables) were used to compare patients between cardiotoxicity status

other cancers (4.5 vs 3.9, P value 0.25 , for breast cancer and 3.3 vs 3.9 , $P$ value 0.26 , for all other cancers).

\section{Decreased versus normal baseline GLS}

Patients with an decreased baseline GLS were most likely male ( $43.94 \%$ vs $27.05 \%, p=0.023)$, had a higher BMI (30.03 \pm 5.99 vs $27.53 \pm 5.96, p=0.007)$, and had a lower baseline LVEF $(62.39 \% \pm 4.43 \%$ vs. $65.01 \% \pm 3.32 \%$, $p<0.001)$. There were no differences in age, comorbidities, baseline blood pressure and heart rate (by echocardiogram report) or cancer type (Table 2).

\section{Univariable and multivariable analyses}

Median time to $>10 \%$ reduction in LVEF was 107 days (range 12.5-729). In a univariable Cox proportional hazards regression analysis, baseline GLS as continuous variable or categorical variable was significantly associated with CTRD (HR $=1.20$, 95\% CI: 1.05, 1.38; $p=0.01$ for continuous and $\mathrm{HR}=4.44,95 \% \mathrm{CI}: 1.83,10.8 ; p<0.001$ as categorical). In addition to GLS, age, male gender, baseline systolic pressure, baseline LVEF, history of hypertension and tobacco use, breast cancer, and cumulative chemotherapy dose were found significant in the univariable model for CTRD. In the multivariable analysis, baseline GLS as continuous variable or decreased GLS $(\geq(-18 \%))$, baseline systolic pressure, history of tobacco use, and chemotherapy dose were significant (Table 3). The final models fitted well (the p-values for the HosmerLemeshow type goodness-of-fit from likelihood-ratio test were $p=0.79$ when GLS as continuous variable and $p=$ 0.51 when GLS as categorical variable).

The optimal cutoff point for GLS at baseline with CTRD was $-18.05 \%$. The sensitivity was 0.70 and specificity was 0.70 . The area under ROC curve was 0.70 . 
Table 2 Baseline characteristic by baseline GLS [Decreased GLS defined as GLS $\geq(-18 \%)$ ]

\begin{tabular}{|c|c|c|c|c|}
\hline & \multirow{2}{*}{$\begin{array}{l}\text { Total } \\
N=188\end{array}$} & \multicolumn{2}{|c|}{ Decreased Baseline GLS } & \multirow{2}{*}{$\begin{array}{l}P \text { - } \\
\text { value }\end{array}$} \\
\hline & & $\begin{array}{l}\text { No } \\
N=122\end{array}$ & $\begin{array}{l}\text { Yes } \\
N=66\end{array}$ & \\
\hline Age (years) & $53.89 \pm 14.40$ & $53.62 \pm 14.86$ & $54.38 \pm 13.60$ & 0.73 \\
\hline Male & $62(32.98)$ & $33(27.05)$ & $29(43.94)$ & 0.023 \\
\hline $\mathrm{BMI}\left(\mathrm{kg} / \mathrm{m}^{2}\right)$ & $28.40 \pm 6.08$ & $27.53 \pm 5.96$ & $30.03 \pm 5.99$ & 0.007 \\
\hline Race & & & & 0.91 \\
\hline Asian & $5(2.66)$ & $4(3.28)$ & $1(1.52)$ & \\
\hline Black & $24(12.77)$ & $14(11.48)$ & $10(15.15)$ & \\
\hline Caucasian & $99(52.66)$ & $65(53.28)$ & $34(51.52)$ & \\
\hline Hispanic & $50(26.60)$ & $33(27.05)$ & $17(25.76)$ & \\
\hline Other & $10(5.32)$ & $6(4.92)$ & $4(6.06)$ & \\
\hline Systolic & $129.42 \pm 17.68$ & $127.97 \pm 16.42$ & $132.17 \pm 19.69$ & 0.13 \\
\hline Diastolic & $70.97 \pm 10.66$ & $69.97 \pm 9.84$ & $72.86 \pm 11.91$ & 0.083 \\
\hline Heart Rate & $76.38 \pm 13.31$ & $76.06 \pm 12.80$ & $76.95 \pm 14.27$ & 0.68 \\
\hline Family History of Heart Disease & $19(10.11)$ & $13(10.66)$ & $6(9.09)$ & 0.81 \\
\hline Diabetes & $35(18.62)$ & $19(15.57)$ & $16(24.24)$ & 0.17 \\
\hline Hypertension & $80(42.55)$ & $50(40.98)$ & $30(45.45)$ & 0.64 \\
\hline Hyperlipidemia & $52(27.66)$ & $35(28.69)$ & $17(25.76)$ & 0.73 \\
\hline Coronary Artery Disease & $9(4.79)$ & $4(3.28)$ & $5(7.58)$ & 0.28 \\
\hline Hypothyroidism & $21(11.17)$ & $16(13.11)$ & $5(7.58)$ & 0.33 \\
\hline Smoking & $48(25.53)$ & $27(22.13)$ & $21(31.82)$ & 0.16 \\
\hline Ejection Fraction & $64.09 \pm 3.94$ & $65.01 \pm 3.32$ & $62.39 \pm 4.43$ & $<0.001$ \\
\hline Cancer type & & & & 0.24 \\
\hline Breast & $80(42.55)$ & $57(46.72)$ & $23(34.85)$ & \\
\hline Hematologic & $99(52.66)$ & $60(49.18)$ & $39(59.09)$ & \\
\hline Other & $9(4.79)$ & $5(4.10)$ & $4(6.06)$ & \\
\hline Chemotherapy dose & $168.70 \pm 102.91$ & $165.56 \pm 95.49$ & $174.27 \pm 115.47$ & 0.58 \\
\hline
\end{tabular}

Data were presented as mean \pm SD for continuous variables and number (\%) for categorical variables. Chi-square or Fisher's exact test (categorical variables) and ttest or Mann-Whitney test (continuous variables) were used to compare patients between abnormal and normal GLS

There was no significant difference in overall all-cause mortality between patients with decreased vs. normal baseline GLS strain ( $\log$-rank $p=0.26)$. The $\mathrm{p}$ values for the survival comparison between GLS $\geq(-18 \%)$ vs GLS< $(-18 \%)$ are 0.67 at 1 year, 0.33 at 3 years and 0.13 at 5 years, respectively (Fig. 2, Table 4). However, CV-related mortality was significantly higher in the decreased GLS $>-18 \%$ group (Log-rank $p<0.001$ ) (Fig. 3).

\section{Discussion}

In this study, we found that patients with normal LVEF and decreased baseline GLS were more likely not only to develop CTRD, defined by a drop of $\mathrm{EF}>10 \%$ points and to final value $<50 \%$, but these patients with baseline GLS abnormalities also had a statistically significant increase in CV-related mortality. In fact, a baseline GLS>-18\% was associated with a greater than 4-fold increased risk of CTRD. Furthermore, there was an increased risk of CTRD in the group with an abnormal baseline GLS despite a lower cumulative anthracycline dose in this group compared to controls.

The American Society of Clinical Oncology (ASCO) recommends heightened monitoring for patients at higher risk of CTRD, and these risk factors include higher dose, concomitant her-2 antagonists or chest radiation, age, traditional cardiac risk factors, and prior myocardial infarction. In addition, compromised cardiac function by imaging --specifically valvular disease or LVEF $<55 \%$-- are considered risk factors. The use of baseline strain assessment by itself as a risk assessment (as opposed to its measurement only to establish a baseline to measure subsequent relative reduction in GLS) is not currently in the guidelines and our study brings additional awareness to emerging data that shows that consideration should be given to the inclusion of baseline GLS as risk factor by itself. 
Table 3 Hazards ratio (HR) and 95\% Cl for developing cardiotoxicity

\begin{tabular}{|c|c|c|c|c|}
\hline & \multicolumn{2}{|l|}{ Univariable } & \multicolumn{2}{|l|}{ Multivariable } \\
\hline & HR $(95 \% \mathrm{Cl})$ & $p$-value & HR (95\% CI) & $p$-value \\
\hline GLS & $1.20(1.05,1.38)$ & 0.01 & $1.17(1.00,1.36)$ & 0.044 \\
\hline GLS$[\geq(-18 \%)$ vs $<(-18 \%)]$ & $4.44(1.83,10.8)$ & $<0.001$ & $3.54(1.34,9.35)$ & 0.011 \\
\hline Age & $1.04(1.01,1.07)$ & 0.01 & & \\
\hline Male & $2.56(1.13,5.8)$ & 0.02 & & \\
\hline BMl & $1.01(.94,1.08)$ & 0.87 & & \\
\hline Systolic & $1.03(1,1.05)$ & 0.02 & $1.03(1.01,1.05)$ & 0.012 \\
\hline HR & $1.01(.97,1.04)$ & 0.7 & & \\
\hline LVEF & $0.85(.76,94)$ & $<0.001$ & & \\
\hline Caucasian & $1.67(.71,3.94)$ & 0.24 & & \\
\hline Family $\mathrm{Hx}$ of heart disease & $1.99(.68,5.86)$ & 0.21 & & \\
\hline Diabetes & $1.76(.69,4.49)$ & 0.23 & & \\
\hline HTN & $3.55(1.46,8.63)$ & 0.01 & & \\
\hline Hyperlipidemia & $1.51(.64,3.56)$ & 0.35 & & \\
\hline CAD & $1.03(.14,7.72)$ & 0.97 & & \\
\hline Hypothyroidism & $0.34(.05,2.53)$ & 0.29 & & \\
\hline Smoking & $6.86(2.9,16.21)$ & $<0.001$ & $5.29(2.09,13.39)$ & $<0.001$ \\
\hline Breast cancer & $0.24(.08,69)$ & 0.01 & & \\
\hline Chemotherapy dose & $0.99(.99,1)$ & 0.01 & $0.994(0.989,0.999)$ & 0.018 \\
\hline
\end{tabular}

Multivariable analysis included GLS and variables selected from stepwise selection procedure. The full model included all variables with $p$-value $<0.05$ from univariable analysis. The HR $(95 \% \mathrm{Cl})$ were presented when $\mathrm{GLS}$ was modeled as continuous variable. Then the same multivariable analysis was done when GLS modeled as a categorical variable [defined as $\geq(-18 \%)$ vs $<(-18 \%)$ ]

Beyond the established risk factors including cumulative anthracycline dose, it is unclear why some patients without these risk factors develop CTRD. There is recent

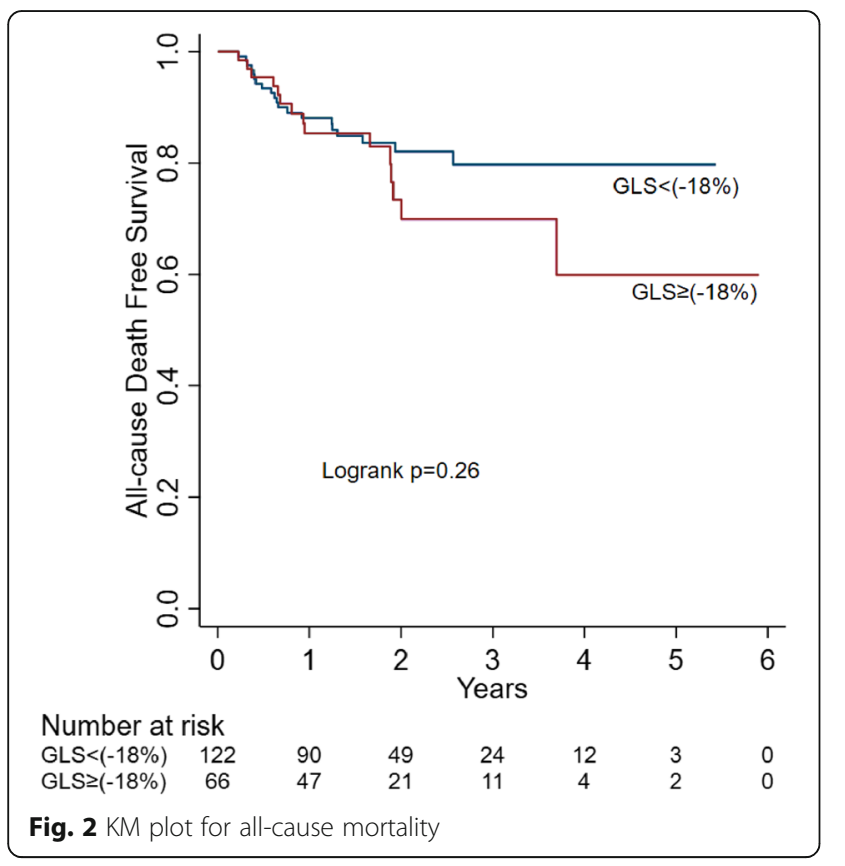

preliminary evidence that some of these patients may be genetically predisposed. A recent study of 213 chemotherapy cardiomyopathy patients from several cohorts found $26(12 \%)$ had genetic variants, predominantly truncating variants in the titin gene (TTN), that are known to cause familial dilated cardiomyopathy. Furthermore, a mouse model with TTN mutations only developed heart failure if they were also treated with anthracyclines while wild type mice treated with the same dose did not develop heart failure. This reinforces the idea that some patients may be predisposed to develop cardiomyopathy and that chemotherapy is the "second hit" that causes cardiomyopathy. Similarly, baseline abnormalities in strain may reflect a structurally abnormal heart that is more susceptible to chemotherapy.

Changes in GLS in patients while receiving anthracycline-based chemotherapy is a well-established predictor of CTRD. The role of baseline GLS is certainly

Table 4 Survival probability at different time point by GLS

\begin{tabular}{llll}
\hline & GLS $<(-18 \%)$ & GLS $\geq(\mathbf{- 1 8 \% )}$ & $P$-value \\
\hline 1 year & $0.87(0.80-0.92)$ & $0.85(0.74-0.92)$ & 0.67 \\
3 year & $0.79(0.69-0.86)$ & $0.71(0.54-0.83)$ & 0.33 \\
5 year & $0.79(0.69-0.86)$ & $0.62(0.39-0.79)$ & 0.13 \\
\hline
\end{tabular}




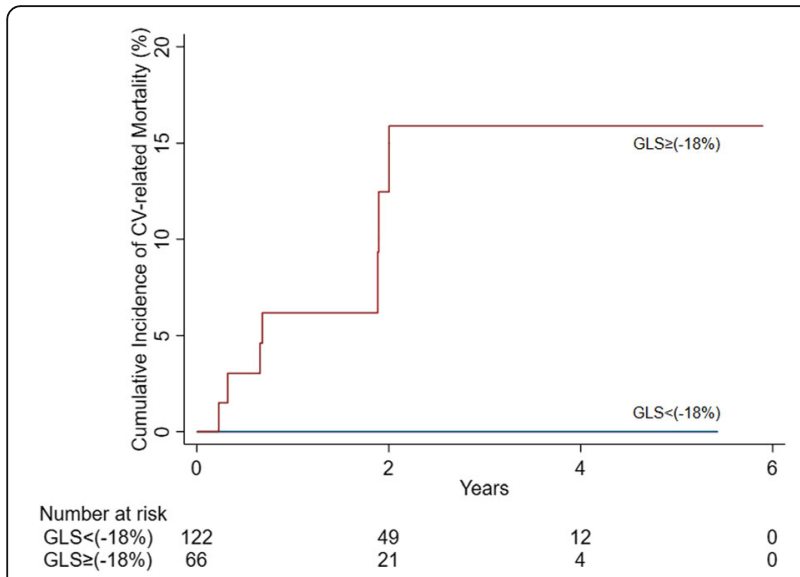

Fig. 3 Cumulative incidence of CV-related mortality adjusted for competing risk of non-cardiovascular death

less established, but other studies have also found that it may predict CTRD. Our study has two important distinguishing features from the prior studies. First, most of these studies have only been in patients with hematological malignancies, which have a higher upfront anthracycline exposure. This is the first study to date to our knowledge evaluating decreased baseline GLS in a broad cohort of cancer patients with lower pretreatment risk of developing anthracycline CTRD per ASCO. Patients with hematological malignancies were more likely to have CTRD than breast cancer patients. The second notable difference is that our study is the only, to our knowledge, to exclude patients with borderline LVEF. Our study did not include anyone with an EF $<55 \%$, thus affirming that decreased baseline GLS has clinical utility in predicting anthracycline CTRD in patients with normal LVEF. One study found an abnormal baseline GLS (cutoff defined as >-15) to be the highest predictive factor in their risk score of patients with acute leukemia. Importantly, 30 of the 450 patients in this study had an LVEF $<53 \%$ at baseline and this characteristic was included in their risk score [9].

In addition, decreased GLS was associated with an increase in CV mortality. This reinforces findings from other anthracycline studies. In a study of 450 patients with hematological malignancies, baseline GLS >-17.5 was associated with a combination of cardiac death or symptomatic heart failure [7]. Although confounded by inclusion of patients with a borderline LVEF 50-55\%, Kang et al. found that a GLS> -15 was associated with all-cause mortality [9]. Currently, abnormal GLS in and of itself is not established in guidelines to represent Stage B heart failure, although there is evidence and debate in favor of the inclusion $[15,16]$. Our study adds additional support that decreased GLS represents may represent microstructural changes in myocardium with significant prognostic implications.
In addition to highlighting the increased risk associated with abnormal baseline GLS abnormalities, it remains unknown if that risk can be mitigated by cardioprotective strategies, Recently, for the first time, the use of strain to guide clinical decision making has been studied, demonstrating that using cardioprotective medications for patients with a change in strain $(12 \%$ relative reduction) during chemotherapy can reduce the incidence of CTRD. Future studies should investigate cardioprotective strategies in patients with baseline GLS abnormalities [17].

\section{Study limitations}

First, this is a single center study that was performed retrospectively. Another limitation is that even though patients with an LVEF $<55 \%$ were excluded from the study, there was a small but statistically significant lower baseline LVEF in the CTRD group. However, decreased baseline GLS was more predictive than LVEF in predicting CTRD, reinforcing the concept that baseline GLS has additional utility in identifying patients at low pretreatment risk of anthracycline CTRD. Finally, there were important baseline differences and thus potential confounding factors between the groups, including higher blood pressure, history of hypertension, increased age and more prevalent smoking history in the CTRD group. Regardless, baseline GLS was more predictive of each of these factors in the multivariate analysis.

\section{Conclusion}

Baseline GLS has predictive value in identifying patients with low-to-moderate pre-treatment risk of anthracycline CTRD. Furthermore, decreased baseline GLS may be associated with increased CV mortality among patients receiving anthracyclines. The results of this study favor broader implementation of STE in cardio-oncology practice for thorough risk stratification and identification of patients at higher risk for developing anthracyclineinduced CTRD than indicated by pre-treatment comorbidities and cancer therapy related risk factors.

\section{Abbreviations}

STE: 2-dimensional speckle tracking strain protocol echocardiography; BMI: Body mass index; CABG: Coronary artery bypass grafting; GLS: Global longitudinal strain; LVEF: Left ventricular ejection fraction

\section{Acknowledgements}

Not applicable.

\section{Disclosures}

The authors have no relevant disclosures.

\section{Authors' contributions}

$\mathrm{RA}, \mathrm{KC}$, and $\mathrm{YC}$ retrospectively collected and analyzed all clinical data. JX performed statistical analyses. AS and CB analyzed echocardiogram images and data. RA and BT were major contributors in writing the manuscript. EK, MK and JX contributed to manuscript writing and editing. BT verified and supervised all the findings of this work. All authors discussed the results and 
contributed to the final manuscript. All authors read and approved the final manuscript.

\section{Funding}

Internal funding sources were used for the purpose of this study.

\section{Availability of data and materials}

The datasets used and/or analyzed during the current study are available from the corresponding author on reasonable request.

\section{Ethics approval and consent to participate}

This study was performed under our Institutional Review Board Approval.

\section{Consent for publication}

Consent to participate and consent for publication were not applicable to this retrospective chart review study.

\section{Competing interests}

The authors declare that they have no competing interests. The authors report no disclosures or conflicts of interest.

\section{Author details}

'Houston Methodist DeBakey Heart and Vascular Center, 6550 Fannin St., Suite 1901, Houston, Texas 77030, USA. ${ }^{2}$ Department of Medicine, Division of Cardiovascular Medicine, University of Missouri-Columbia, Columbia, Missouri, USA. ${ }^{3}$ Center for Outcomes Research, Houston Methodist Research Institute, Houston, Texas, USA.

Received: 27 September 2020 Accepted: 19 January 2021

Published online: 31 January 2021

\section{References}

1. Armenian $\mathrm{SH}$, Lacchetti C, Barac A, et al. Prevention and monitoring of cardiac dysfunction in survivors of adult cancers: American Society of Clinical Oncology Clinical Practice Guideline. J Clin Oncol. 2017;35:893-911.

2. Thavendiranathan P, Poulin F, Lim KD, Plana JC, Woo A, Marwick TH. Use of myocardial strain imaging by echocardiography for the early detection of cardiotoxicity in patients during and after cancer chemotherapy: a systematic review. J Am Coll Cardiol. 2014;63:2751-68.

3. Charbonnel C, Convers-Domart R, Rigaudeau S, et al. Assessment of global longitudinal strain at low-dose anthracycline-based chemotherapy, for the prediction of subsequent cardiotoxicity. Eur Heart J Cardiovasc Imaging. 2017;18:392-401.

4. Oikonomou EK, Kokkinidis DG, Kampaktsis PN, et al. Assessment of prognostic value of left ventricular global longitudinal strain for early prediction of chemotherapy-induced cardiotoxicity: a systematic review and meta-analysis. JAMA Cardiol. 2019;4:1007-18.

5. Plana JC, Galderisi M, Barac A, et al. Expert consensus for multimodality imaging evaluation of adult patients during and after cancer therapy: a report from the American Society of Echocardiography and the European Association of Cardiovascular Imaging. J Am Soc Echocardiogr. 2014;27:91139.

6. Seidman A, Hudis C, Pierri MK, et al. Cardiac dysfunction in the trastuzumab clinical trials experience. J Clin Oncol. 2002;20:1215-21.

7. Ali MT, Yucel E, Bouras $\mathrm{S}$, et al. Myocardial strain is associated with adverse clinical cardiac events in patients treated with anthracyclines. J Am Soc Echocardiogr. 2016:29:522-527.e3.

8. Hatazawa K, Tanaka H, Nonaka A, et al. Baseline global longitudinal strain as a predictor of left ventricular dysfunction and hospitalization for heart failure of patients with malignant lymphoma after anthracycline therapy. Circ J. 2018:82:2566-74

9. Kang $Y$, Assuncao BL, Denduluri S, et al. Symptomatic heart failure in acute leukemia patients treated with anthracyclines. JACC CardioOncol. 2019;1: 208-17.

10. Zamorano JL, Lancellotti P, Rodriguez Munoz D, et al. 2016 ESC position paper on cancer treatments and cardiovascular toxicity developed under the auspices of the ESC Committee for Practice Guidelines: the task force for cancer treatments and cardiovascular toxicity of the European Society of Cardiology (ESC). Eur Heart J. 2016;37:2768-801.

11. Ewer MS. Cancer and the heart. 3rd ed. Raleigh: PMPH USA; 2019. p. 1. online resource
12. Voigt JU, Pedrizzetti G, Lysyansky P, et al. Definitions for a common standard for 2D speckle tracking echocardiography: consensus document of the EACVI/ASE/Industry Task Force to standardize deformation imaging. J Am Soc Echocardiogr. 2015;28:183-93.

13. Liu JE, Barac A, Thavendiranathan P, Scherrer-Crosbie M. Strain imaging in cardio-oncology. JACC CardioOncol. 2020;2:677-89.

14. Balakrishnan N, Rao CR. Advances in survival analysis, vol. 23. 1st ed. Amsterdam; Boston: Elsevier North-Holland; 2004. p. 383-94.

15. Wang $Y$, Yang $H$, Huynh Q, Nolan M, Negishi K, Marwick TH. Diagnosis of nonischemic stage B heart failure in type 2 diabetes mellitus: optimal parameters for prediction of heart failure. JACC Cardiovasc Imaging. 2018; 11:1390-400.

16. Kale P, Afzal A. Stage B heart failure: to strain or not to strain. JACC Cardiovasc Imaging. 2018;11:1401-4.

17. Negishi $T$, Thavendiranathan $P$, Penicka $M$, et al. Cardioprotection using strain-guided management of potentially cardiotoxic cancer therapy: 1 year results of the SUCCOUR trial. Eur Heart J. 2020;41(Supplement_2):ehaa9463282.

\section{Publisher's Note}

Springer Nature remains neutral with regard to jurisdictional claims in published maps and institutional affiliations.
Ready to submit your research? Choose BMC and benefit from:

- fast, convenient online submission

- thorough peer review by experienced researchers in your field

- rapid publication on acceptance

- support for research data, including large and complex data types

- gold Open Access which fosters wider collaboration and increased citations

- maximum visibility for your research: over $100 \mathrm{M}$ website views per year

At BMC, research is always in progress.

Learn more biomedcentral.com/submissions 